\title{
Proposing a Core Outcome Set for Physical Activity and Exercise Interventions in People With Rare Neurological Conditions
}

\author{
Gita Ramdharry ${ }^{1,2+}$, Valentina Buscemi ${ }^{1,2}$, Annette Boaz ${ }^{3}$, Helen Dawes ${ }^{4}$, Thomas Jaki ${ }^{5,6}$, \\ Fiona Jones ${ }^{3,7}$, Jonathan Marsden ${ }^{8}$, Lorna Paul ${ }^{9}$, Rebecca Playle ${ }^{10}$, Elizabeth Randell ${ }^{10}$, \\ Michael Robling ${ }^{10}$, Lynn Rochester ${ }^{11}$ and Monica Busse ${ }^{10 *+}$ \\ ${ }^{1}$ National Hospital for Neurology and Neurosurgery, University College London Hospitals NHS Trust, London, \\ United Kingdom, ${ }^{2}$ Institute of Neurology, University College London, London, United Kingdom, ${ }^{3}$ Faculty of Health, Social \\ Care and Education, St. George's, University of London, London, United Kingdom, ' ${ }^{4}$ Faculty of Health and Life Sciences, \\ Oxford Brookes University, Oxford, United Kingdom, ${ }^{5}$ Medical Research Council Biostatistics Unit, University of Cambridge, \\ Cambridge, United Kingdom, ${ }^{6}$ Department of Mathematics and Statistics, University of Lancaster, Lancaster, \\ United Kingdom, ${ }^{7}$ Faculty of Health, Social Care and Education, Kingston University, Surrey, United Kingdom, ${ }^{8}$ Faculty of \\ Health, Plymouth University, Plymouth, United Kingdom, ${ }^{9}$ Department of Physiotherapy and Paramedicine, Glasgow \\ Caledonian University, Glasgow, United Kingdom, ${ }^{10}$ Centre for Trials Research, Cardiff University, Cardiff, United Kingdom, \\ ${ }^{11}$ Institute of Neuroscience, Newcastle University, Newcastle, United Kingdom
}

OPEN ACCESS

Edited by

Jennifer Ryan,

Royal College of Surgeons in

Ireland, Ireland

Reviewed by:

Paulo Henrique Silva Pelicioni, University of Otago, New Zealand Aoife Stephenson,

Royal College of Surgeons in Ireland, Ireland

Patrick McPhee

McMaster University, Canada

*Correspondence: Monica Busse busseme@cardiff.ac.uk

tThese authors have contributed equally to this work

Specialty section:

This article was submitted to Disability, Rehabilitation, and Inclusion,

a section of the journal

Frontiers in Rehabilitation Sciences

Received: 05 May 2021 Accepted: 23 September 2021

Published: 21 October 2021

Citation

Ramdharry G, Buscemi V, Boaz A, Dawes $H$, Jaki $T$, Jones F, Marsden J, Paul L, Playle R, Randell E, Robling M,

Rochester $L$ and Busse M (2021)

Proposing a Core Outcome Set for

Physical Activity and Exercise Interventions in People With Rare

Neurological Conditions. Front. Rehabilit. Sci. 2:705474. doi: 10.3389/fresc.2021.705474
Rare neurological conditions (RNCs) encompass a variety of diseases that differ in progression and symptoms but typically include muscle weakness, sensory and balance impairment and difficulty with coordinating voluntary movement. This can limit overall physical activity, so interventions to address this are recommended. The aim of this study was to agree a core outcome measurement set for physical activity interventions in people living with RNCs. We followed established guidelines to develop core outcome sets. Broad ranging discussions in a series of stakeholder workshops led to the consensus that (1) physical well-being; (2) psychological well-being and (3) participation in day-to-day activities should be evaluated in interventions. Recommendations were further informed by a scoping review of physical activity interventions for people living with RNCs. Nearly 200 outcome measures were identified from the review with a specific focus on activities or functions (e.g, on lower limb function, ability to perform daily tasks) but limited consideration of participation based outcomes (e.g., social interaction, work and leisure). Follow on searches identified two instruments that matched the priority areas: the Oxford Participation and Activities Questionnaire and the Sources of Self-Efficacy for Physical Activity. We propose these scales as measures to assess outcomes that are particularly relevant to assess when evaluating physical activity interventions mong people with RNCs. Validation work across rare neurological conditions is now required to inform application of this core outcome set in future clinical trials to facilitate syntheses of results and meta-analyses.

Keywords: physical activity, neuromuscular disease, motor neurone disease, Huntington's disease, inherited ataxias, hereditary spastic paraplegia, parkinsonism, outcome measurement instruments 


\section{INTRODUCTION}

Rare neurological conditions (RNCs), where cases are $\leq 40$ per 100,000 population (1), collectively incur a significant cost burden to healthcare, social care services and informal care (2). Despite variability across conditions, many of these conditions will share symptoms and signs at the level of body function, activities and participation (3). As such, common approaches to improve fitness (e.g., cardiovascular and strength training), activity (e.g., balance and gait training) and participation levels (e.g., supported self-management) are often implemented in clinical practice (4-8).

Physical activity is any bodily movement produced by the muscles that require us to expend energy and can include structured exercise, active transportation, household chores, and activity during work, play and recreation (9). Whilst trials of physical activity interventions in RNCs highlight the potential of physical activity interventions to improve fitness and function (10-13) they are often small studies that fail to influence clinical practice. There are a variety of factors that limit the impact of these trials, not least the selection of outcome measures. Measurement constructs may vary from physiological measures (e.g., strength and fitness), to functional assessments (e.g., walking speed, climbing stairs) or quality of life and wellbeing outcomes and do not typically take into account patient preferences (14). If we are to ensure that research is relevant and able to influence clinical practice and future research, we need to ensure the use (and reporting) of standardized, relevant outcome measures within the field that are applicable to people living with the conditions $(15,16)$. Importantly, it should not be assumed that measurement should be restricted to the agreed core outcomes but rather that these outcomes should always be gathered and reported to facilitate evidence synthesis across relevant trials and studies. Core outcome sets have been agreed for specific target conditions for example cancer, rheumatology and chronic pain as well as for specific care pathways for example maternity care $(17,18)$.

Core outcome sets have also been proposed for people with neurologic conditions (19), including adults with dementia (20). However, these core sets have not been tailored to physical activity interventions for people living with RNCs. Recommending measures would not only help to bring consistency in reporting, allowing comparisons or meta-analysis of future studies, but also ensure responses are measured of constructs important to people living with RNCs. This study focuses on the development of an agreed standardized set of outcomes termed a "core outcome set" (21) that should be at a minimum measured and reported in trials of physical activity interventions in people living with RNCs.

\section{METHOD}

The study followed the guidelines of the COSMIN (COnsensusbased Standards for the selection of health Measurement INstruments) and COMET (Core Outcome Measures in Effectiveness Trials) initiatives. A four step approach was used to select outcome measurement instruments recommended within core outcome sets (21). The activities relevant to each step within the process are described in detail below.

We focused on groups of progressive RNCs, namely neuromuscular diseases, Ataxias, Huntington's Disease (HD), Atypical Parkinson Diseases (AP), including Progressive Supranuclear Palsy, Multiple Systems Atrophy, Corticobasal Degeneration, Motor Neuron Diseases (MND) and Hereditary Spastic Paraparesis (HSP). These conditions affect $\sim 2-10$ per 100,000 in the general population, collectively leading to limited mobility and poor balance for many individuals. People with neuromuscular diseases and MND experience profound weakness and muscle atrophy. People with Ataxia, HD, AP, and HSP experience difficulty controlling movement, with some muscle weakness and variable cognitive impairment. Many people across the conditions also experience pain, joint deformity, fatigue and depression which impacts on their ability to participate in routine activities of daily living.

\section{Step One: Conceptual Considerations}

People living with RNCs, carers of people with RNCs and representatives from five collaborating support groups and charities, namely the Muscular Dystrophy Association, Ataxia UK, HSP support group, PSP Association, HD Association of England and Wales were invited to join a stakeholder group. They attended an initial workshop (Workshop 1) to define conceptual considerations in relation to the physical activity interventions and outcomes in our target population, namely, people living with RNCs.

This was followed by a second stakeholder workshop (Workshop 2) with people living with RNC and the relevant charity representatives. They worked together to (a) explore issues and experiences relating to physical activity in the face of living with a RNC and (b) identify and priorities key constructs and domains of importance that would need to be measured when evaluating a physical activity intervention. Representatives from RNC charities were asked to gather views from their members living with RNCs prior to the meeting through their communication channels, e.g., surveys and social media platforms.

\section{Step Two: Finding Existing Outcome Measurement Instruments}

We conducted a scoping review of systematic reviews published between January 2008 and December 2018 to identify outcome measures used to measure efficacy of any type of physical activity intervention for adults with neuromuscular diseases, motor neurone disease (MND), HD, PSP, multiple system atrophy (MSA), inherited ataxias and HSP (Open Science Framework registration: https://osf.io/4cr32/). The research team, experts in this field, were aware that little research into physical activity had taken place until the early 2000s and reviews came later, hence the 10-year window. Studies were included if participants were adults and if the reviews reported at least one outcome measure to evaluate the efficacy of the physical activity intervention at either the body structure/function, activity and/or participation levels, according to the International Classification of Functioning, Disability and Health (ICF) (20). Constructs and domains of 
importance identified during Workshop 2, were matched with outcome measures identified in the scoping review. Where no measures matched the identified, domains, additional literature searches were done using the domain descriptions as search terms. Additional criteria for selection were use in RNC or other neurological diseases. Two of the descriptors, motivation and confidence, relate strongly to self-efficacy so this was also added as a search term. Elicitation of stakeholder opinions during a series of virtual meetings was undertaken to supplement this process and to identify proposed outcome measures that were consistent with the domains of importance identified by the stakeholder group.

\section{Step Three: Quality and Feasibility Assessment of Outcome Measurement Instruments}

Outcome measurement instruments that are included in a core outcome set should ideally be reliable and valid for use in the target populations (22). Feasibility of use is a further consideration. The rarity of the diseases being studied meant that the psychometric properties of the measurement tools we identified had not been examined in these conditions. We thus considered the psychometric properties of the tools as applied to more common long term, neurological conditions where there were indications of some common impairments. The evidence for each was collated for presentation at step four.

\section{Step Four: Reaching Consensus on the Proposed Core Outcome Set}

The outcome measure instruments under scrutiny matching the agreed domains of importance were examined by individual researchers then presented to the wider research team for technical discussions of the psychometric properties through a series of video meetings. Following the video meetings, lists of items assessed within each instrument were sent to the stakeholder group via e-mail to elicit further reflection on their relevance to the constructs of importance. A final face to face stakeholder workshop (workshop 3) and consensus procedure was undertaken to agree on the instruments for each outcome to be recommended for inclusion in the core outcome set.

\section{RESULTS}

\section{Step One: Conceptual Considerations}

People living with RNCs (workshop $1 \mathrm{~N}=5$; workshop $2 \mathrm{~N}$ = 3), carers (workshop $1 N=1$ ) and charity representatives (workshop $1 N=5$; workshop $2 N=5$ ) considered it important that measurement tools were able to detect outcomes across domains of (A) function and well-being and (B) participation in activities. In terms of (A), staying well, ensuring good sleep and maintaining positive mood were of highest priority whilst in relation to (B), the ability and confidence to take control and make choices along with normalization of participation and social engagement were important. Through further discussion, the stakeholder groups agreed that these aspects were well-centered around (i) physical well-being; (ii) psychological well-being and (iii) participation in day-to-day activities as the primary domains of meaningful importance. Relevant constructs within the physical domain were physical function and independence. Constructs in the psychological wellbeing domain were emotional well-being, mood, enjoyment, motivation for physical activity and confidence, whilst leisure activities, work and activity that matters (personal choice) were constructs of importance within the domain of participation.

\section{Step Two: Finding Existing Outcome Measurement Instruments}

Database searches identified 5,435 articles, and, after removing duplicates, 4,433 were screened by titles and abstracts, leaving 62 articles for full-text eligibility assessment. They were screened and 27 were included in the scoping review $(4,5,8,12,13$, 23-44). The results of the scoping review will be presented in detail separately. Nearly 200 outcome measures assessing outcomes of structured physical activity interventions (Table 1) were identified within these 27 articles. Dosage, intensity and duration of training regimes were highly variable but typically involved strength training, aerobic and respiratory, functional training and combined programs with very few focusing on physical activity behavior change.

We mapped each outcome to the World Health Organisation International Classification of Function (ICF) domains (20) (see Table 2). The majority were related to function and activity. Outcomes reflective of both body structure impairments and participation were less frequently reported. Two domains were categorized as "Other" (e.g., Goal attainment score), and "Disease-specific" questionnaires (e.g., Unified Huntington's Disease Rating Scale or Scale for the Assessment and Rating of Ataxia).

Eleven reviews utilized disease-specific outcome measures, while in six reviews measures were not able to be represented within the ICF domains (i.e., in the "Other" category). Most studies $(n=17)$ included outcomes that were representative of three to five different domains. Notably, there was no evidence of stakeholder engagement or involvement of people with the condition being investigated in the selection of measures used as primary outcomes.

Constructs relevant to the physical and psychological wellbeing, and participation in day-to-day activities domains were cross-checked with the outcomes synthesized in the scoping review. No single outcome measurement instrument that addressed all three domains was identified. Measures were usually tailored to specific activities or functions (e.g., on lower limb function, ability to perform daily tasks). Alternative outcomes reflective of wellbeing were reviewed by stakeholders through a series of group discussions. None of these comprehensively matched the domains of importance identified in Step One.

Further literature searching resulted in the Oxford Participation and Activities Questionnaire (Ox-PAQ) $(50,51)$ being identified as an instrument that matched the majority of the constructs highlighted as relevant in 
TABLE 1 | Characteristics (including outcome measures utilized) reported in studies included in scoping reviews of physical activity interventions in RNCs.

\begin{tabular}{|c|c|c|c|c|c|}
\hline References & $\begin{array}{l}\text { Research designs of } \\
\text { included studies }\end{array}$ & $\begin{array}{l}\text { Number of } \\
\text { participants }\end{array}$ & Participant characteristics & Controls & Outcome measures \\
\hline Cup et al. (24) & $\begin{array}{l}2 \text { studies using } \\
\text { pre-post design }\end{array}$ & 17 & $\begin{array}{l}\text { Male/female: } 7 / 10 \\
\text { Mean Age (Y): } \\
\text { Study 1: } 52.4 \text { (range: } 28-67) \\
\text { Study 2: } 62.5 \text { (range: } 39-83 \text { ) }\end{array}$ & No controls & $\begin{array}{l}\text { Performance and satisfaction/Muscle strength/Grip force and pinch } \\
\text { grip (Grippit)/Fine motor control (Purdue Pegboard), Range of } \\
\text { Motion (goniometer). Activities of daily living (interview with } \\
\text { ADL-Taxonomy); Life satisfaction (modified Life Satisfaction Checklist) }\end{array}$ \\
\hline Habers and Takken (27) & $\begin{array}{l}2 \text { RCTs, } 1 \text { non-RCT, } 9 \\
\text { uncontrolled studies }\end{array}$ & 161 & $\begin{array}{l}\text { Male, } N=57 ; \text { Females, } N=104 \\
\text { Mean Age }(Y) \text { : range } 40-68\end{array}$ & Not reported & $\begin{array}{l}\text { Disease activity (e.g., serum levels of creatine kinase, aldolase, } \\
\text { cytokines etc.)/Muscle strength/Aerobic fitness/Functional } \\
\text { performance/Functional capacity/Health status/Lung function/Muscle } \\
\text { characteristics/Disease impact/Fatigue }\end{array}$ \\
\hline Quinlivan et al. (13) & $\begin{array}{l}3 \text { non-randomized } \\
\text { studies }\end{array}$ & 27 & $\begin{array}{l}2 \text { out of } 3 \text { studies reported gender: } 9 \\
\text { males and } 9 \text { females in total. Mean Age } \\
\text { (Y): range } 32-61\end{array}$ & $\begin{array}{l}\text { Same training but in healthy } \\
\text { controls, age- and } \\
\text { sex-matched healthy, } \\
\text { sedentary controls } \\
\text { otherwise not specified }\end{array}$ & $\begin{array}{l}\text { Borg rating of perceived exertion/NO2 max/HR/Superficial EMG for } \\
\text { muscle activity and glucose and lactate blood levels/Serum creatine } \\
\text { kinase /Respiratory gas exchange/Cardiac output and serum samples } \\
\text { for lactate and glucose were measured/Needle muscle biopsy of } \\
\text { vastus/Respiratory gas exchange data were collected/NO2 peak, and } \\
\text { gross mechanical efficiency during the constant workload test/HR using } \\
12 \text { lead ECG tracing/Capillary blood samples lactate and ammonia }\end{array}$ \\
\hline Ydemann et al. (28) & $\begin{array}{l}1 \text { Pre-post cohort, } 4 \\
\text { RCTs, } 1 \text { prospective } \\
\text { cohort, } 1 \text { quasi-RCT, } 1 \\
\text { descriptive study }\end{array}$ & 757 & Not reported & $\begin{array}{l}\text { Standard medical therapy, } \\
\text { usual care, daily interruption } \\
\text { of sedation only, general } \\
\text { physiotherapy alone }\end{array}$ & $\begin{array}{l}6 \mathrm{MWD} / \mathrm{MIP} / \mathrm{Isometric} \text { quadriceps force/Subjective feeling of functional } \\
\text { well-being/Time in bed//CU stay/Hospital stay/Duration of } \\
\text { delirium/Ventilator-free days/Muscle fatigue and isotime } \\
\text { dyspnoea/Atrophy/Weaning of atrophy (no further details provided) }\end{array}$ \\
\hline Voet et al. (23) & $\begin{array}{l}4 \mathrm{RCTs} \text { and } 1 \text { quasi } \\
\text { randomized study }\end{array}$ & 170 & $\begin{array}{l}20 \text { adults with mitochondrial myopathy, } \\
\text { diagnosed on the basis of clinical, familial } \\
\text { and muscle biopsy data. } \\
35 \text { adults with myotonic dystrophy type } 1 \text {, } \\
\text { genetically confirmed. } \\
36 \text { adults with myotonic dystrophy (2 } \\
\text { congenital form, } 34 \text { classical adult type), } \\
\text { diagnosis not verified. } \\
65 \text { adults with FSHD, } \\
\text { genetically confirmed. } \\
9 \text { adults with dermatomyositis and } 5 \\
\text { adults with polymyositis. }\end{array}$ & $\begin{array}{l}\text { Strength training vs. no } \\
\text { training }\end{array}$ & $\begin{array}{l}\text { Differences in Muscle strength (using dynamometer)/Quantitative } \\
\text { Muscle Assessment fixed myometry testing system/Dynamic strength } \\
\text { was evaluated using the one repetition maximum / Weight-lifting } \\
\text { capacity/ Endurance time measured in a submaximal cycling test at a } \\
\text { constant workload of } 70 \% / 6 \text { MWT, VO2 max/ Maximum duration of } \\
\text { contraction at } 80 \% \text { of MVIC on an isokinetic dynamometer/Sickness } \\
\text { Impact Profile and the Symptom-Checklist /Nottingham Health Profile/ } \\
\text { SF-36 Health Survey/ CIS-fatigue }\end{array}$ \\
\hline Gianola et al. (25) & $\begin{array}{l}4 \text { ( } 3 \text { controlled and } 1 \\
\text { randomized clinical) } \\
\text { trials. One study was } \\
\text { excluded as included } \\
\text { participants under } 18 \\
\text { years old. }\end{array}$ & 128 & $\begin{array}{l}\text { Mean Age of participants range from } 22 \text { to } \\
48 \text { years }\end{array}$ & $\begin{array}{l}\text { No control/healthy control } \\
\text { group/other interventions }\end{array}$ & $\begin{array}{l}\text { Maximal voluntary isometric contraction /Maximal peak } \\
\text { torque/Modified MRC/Six-Minute Bicycle Test/BORG/Six-minute walk } \\
\text { test (m)/Timed-stands test/Timed up-and-go test/MVIC isokinetic } \\
\text { torque/Test } 80 \% \text { MVC (sec)/Descending stairs/Climbing } \\
\text { stairs/Standing up from a chair/Standing up from lying supine/Walking } \\
6 \text { min (comfortably)/Walking } 50 \mathrm{~m} \text { (fast) (sec)/CIS-fatigue/ICF functional } \\
\text { dimensions }\end{array}$ \\
\hline $\begin{array}{l}\text { Narayanaswami et al. } \\
\text { (26) }\end{array}$ & 5 Class III studies & 62 & $\begin{array}{l}12 \text { patients with Welander distal myopathy } \\
9 \text { ambulatory patients with LGMD2I and } 9 \\
\text { healthy controls } 11 \text { men with BMD and } 7 \\
\text { healthy men } 8 \text { patients with hIBM3 } \\
\text { secondary to a defect in the MYH2 gene } 6 \\
\text { patients with hIBM3 secondary to a defect } \\
\text { in the MYH2 gene }\end{array}$ & $\begin{array}{l}\text { Sedentary, age-matched } \\
\text { controls }\end{array}$ & $\begin{array}{l}\text { Maximal oxygen uptake/Maximal workload, and other patient-reported } \\
\text { outcomes/Maximum workload/Muscle strength/Change in the } \\
\text { expression of myosin isoforms on muscle biopsy }\end{array}$ \\
\hline
\end{tabular}


TABLE 1 | Continued

\begin{tabular}{|c|c|c|c|c|c|}
\hline References & $\begin{array}{l}\text { Research designs of } \\
\text { included studies }\end{array}$ & $\begin{array}{l}\text { Number of } \\
\text { participants }\end{array}$ & Participant characteristics & Controls & Outcome measures \\
\hline Khan and Amatya (32) & $\begin{array}{l}1 \text { SRV, } 1 \text { RCT, } 1 \\
\text { case-control, } 5 \\
\text { prospective or } \\
\text { retrospective cohort } \\
\text { studies, } 6 \text { case } \\
\text { series/reports }\end{array}$ & 422 & Not reported & $\begin{array}{l}\text { Low-intensity home based } \\
\text { program of maintenance } \\
\text { exercises and education for } \\
\text { self-management ( } 30 \text { min } \\
\text { twice a week) (RCT) or } \\
\text { healthy controls. }\end{array}$ & $\begin{array}{l}\text { HRQoL/FIM/PIPPS/DASS/WHOQoL/LOS/Modified Barthel Index/MRS } \\
\text { /HDS NHP/BI/ESS/HAS }\end{array}$ \\
\hline $\begin{array}{l}\text { Simatos Arsenault et al. } \\
\text { (12) }\end{array}$ & $\begin{array}{l}1 \text { single subject, } 4 \text { case } \\
\text { reports, } 1 \text { quasi } \\
\text { experimental design, } 1 \\
\text { RCT }\end{array}$ & 133 & $\begin{array}{l}66 \text { females vs. } 67 \text { males Mean Age } \\
\text { (Y): } 43.8\end{array}$ & $\begin{array}{l}\text { No exercise or lower } \\
\text { intensity, home-based } \\
\text { ambulatory exercise, } \\
\text { otherwise not reported }\end{array}$ & $\begin{array}{l}\text { FSS/Activity monitor/SF-36, FIS/Perceived mental functioning/Physical } \\
\text { fitness (peak work levels, VO2 mL/min, } \\
\mathrm{mL} / \mathrm{kg} / \mathrm{min} \text { )/Nentilation/lsokinetic leg strength (total work } \\
\text { capacity)/General mobility/Confidence in walking/Cardiorespiratory } \\
\text { cycle ergometer test/lsokinetic muscle strength/Functional outcome of } \\
\text { daily physical activity (RAM)/FIS (cognitive, physical, and social)/GBS } \\
\text { disability score/HADS/RHS/QOL } \\
\text { SF-36/MMT/WHOQOL-BREF/DASS-21/PIPP/Physical fitness (duration } \\
\text { of exercise, distance walking, distance cycling, grip } \\
\text { strength)/Pulmonary fitness (PEFR, FVC, FEV1) }\end{array}$ \\
\hline Young et al. (29) & $\begin{array}{l}1 \text { Randomized } \\
\text { controlled single blind } \\
\text { trial }\end{array}$ & 29 & Not reported & No strength training & $\begin{array}{l}\text { Muscle strength voluntary contraction//sokinetic knee torques/Timed } \\
\text { functional activities }\end{array}$ \\
\hline Sman et al. (5) & $\begin{array}{l}3 \text { RCTs, } 5 \\
\text { quasi-experimental } \\
\text { (i.e., pre-post testing), } \\
1 \text { case report }\end{array}$ & 134 & $\begin{array}{l}\text { Average age: } 38 \text { years old. } \\
8 \text { out of } 9 \text { studies reported gender: } 52 \% \\
\text { were male, } 48 \% \text { female. }\end{array}$ & $\begin{array}{l}\text { Where reported: controls } \\
\text { underwent the same } \\
\text { program, however, balance } \\
\text { training was managed by a } \\
\text { physiotherapist instead of a } \\
\text { mechanical apparatus }\end{array}$ & $\begin{array}{l}\text { Muscle strength (N or Kg)/Maximal voluntary isometric testing } \\
\text { (Kg)//sokinetic knee torque flex/extension/MVC/Endurance test at } 80 \% \\
\text { MVC/Isokinetic muscle strength (Nm)/Medical research council scale } \\
\text { (MRC)/BOT (balance) score/Power/Long jump (cm)/6 MWT/Walking } \\
\text { ability (different parameters)/Functional activities (e.g., Chair } \\
\text { raise)/CMTES/Phone FITT FDI/ROM/Tinetti Scale/Berg Balance } \\
\text { scale/Physiological (BMI,FFM, Percent body fat, Serum myoglobin, } \\
\text { RMS ( }(\mu v) \text { /Fatigue Severity Scale/Modified PCI/MHC/Myosin heavy } \\
\text { chain/Cardiorespiratory cycle test/Mean blood CKNAS/NO2 } \\
\text { max/HR/Respiratory Borg Scale/METS/Fatigue Borg Scale }\end{array}$ \\
\hline Corrado et al. (30) & 4 RCTs and 1 Cohort & 236 & Not reported & No intervention & $\begin{array}{l}\text { Quantitative neuromuscular assessment/Bioelectrical impedance } \\
\text { analysis/6 MWT/MVC (myometer or isokynetic dynamometre)/Borg } \\
\text { scale/Serum level of myoglobin/Surface electromyography } \\
\text { techniques/Holter }\end{array}$ \\
\hline Lui and Byl (33) & $\begin{array}{l}\text { Prospective clinical } \\
\text { studies }(N=2), \mathrm{RCTs} \\
(N=2) \text { and } 1 \mathrm{SRV} \\
\text { (Dalbello-Haas et al. } \\
\text { (47), previous version of } \\
\text { Dal Bello and Florence } \\
\text { (4)) }\end{array}$ & $\begin{array}{l}98 \text { (including Drory } \\
\text { et al. (45); } \\
\text { Bello-Haas et al. } \\
\text { (46)), excluding } \\
\text { Dalbello-Haas } \\
\text { et al. (47) }\end{array}$ & Not reported & $\begin{array}{l}\text { Usual care/home exercise } \\
\text { program without } \\
\text { supervision/no exercise } \\
\text { participation or usual } \\
\text { activities }\end{array}$ & Norris ALS score strength/ALS-FRS strength MMT/FSS/FIM/FVC \\
\hline
\end{tabular}




\begin{tabular}{|c|c|c|c|c|c|}
\hline References & $\begin{array}{l}\text { Research designs of } \\
\text { included studies }\end{array}$ & $\begin{array}{l}\text { Number of } \\
\text { participants }\end{array}$ & Participant characteristics & Controls & Outcome measures \\
\hline Ng et al. (37) & $\begin{array}{l}3 \text { prospective studies, } \\
1 \text { cross-sectional, } \\
\text { pre-post case series }\end{array}$ & 779 & Not reported & $\begin{array}{l}\text { General neurology clinic or } \\
\text { general MND care }\end{array}$ & $\begin{array}{l}\text { Survival, hospital readmissions and length of stay, SF-36, VAS on life } \\
\text { satisfaction and well-being, ALSSS, ALSFRS, CSI, healthcare costs }\end{array}$ \\
\hline $\begin{array}{l}\text { Dal Bello-Haas and } \\
\text { Florence (4) }\end{array}$ & $\begin{array}{l}2 \text { studies, } 6 \text { and } 12 \\
\text { month parallel group (1 } \\
\text { randomized and } 1 \\
\text { quasi randomized trial) }\end{array}$ & 52 & $\begin{array}{l}27 \text { people with definite or probable, } \\
\text { probable with laboratory-supported MND } \\
\text { (El Escorial criteria), aged } 41-80 \text { years. } \\
\text { Early stage MND. } 25 \text { people with definite } \\
\text { or probable MND (El Escorial criteria), } \\
\text { aged } 41-80 \text { years. Mild to moderate } \\
\text { stages of MND. }\end{array}$ & $\begin{array}{l}\text { The control condition was } \\
\text { either no exercise or } \\
\text { standard rehabilitation } \\
\text { management (for example, } \\
\text { range of motion exercise or } \\
\text { stretching exercise). }\end{array}$ & $\begin{array}{l}\text { LSFRS/the SF-36 to measure quality of life/FSS/Manual muscle } \\
\text { strength testing }\end{array}$ \\
\hline $\begin{array}{l}\text { Eidenberger and } \\
\text { Nowotny (34) }\end{array}$ & $\begin{array}{l}2 \text { RCTs, } 1 \\
\text { pre-experimental study } \\
\text { and } 1 \text { with a historical } \\
\text { control group }\end{array}$ & 87 & $\begin{array}{l}\text { Male, } N=57 \text {; Females, } N=30 \text { Mean Age } \\
\text { (Y): range 53-63 years }\end{array}$ & $\begin{array}{l}\text { Sham training/historical } \\
\text { controls/no controls/ lowest } \\
\text { possible load }\end{array}$ & $\begin{array}{l}\text { Respiratory-related OMs (e.g., Spirom/FVC/MIP/MEP etc.)/Total } \\
\text { survival time/6 MWT/Hand-held dynamometry/ ALSFRS/ } \\
\text { FSS/HRSD/ESS/FIM/EQ-5D/SF-36/Chronic Respiratory Questionnaire }\end{array}$ \\
\hline $\begin{array}{l}\text { Arbesman and Sheard } \\
\text { (36) }\end{array}$ & $\begin{array}{l}2 \mathrm{RCTs} / 2 \text { non-RCTs }+ \\
\text { single subject study }\end{array}$ & $\begin{array}{l}287 \text { (including Dal } \\
\text { Bello-Haas and } \\
\text { Florence (4)) }\end{array}$ & $\begin{array}{l}\text { Only one study (i.e., single-case study) } \\
\text { reported: one male, age } 62 \text { years. Drory } \\
\text { et al. (45) and Bello-Haas et al. (46) and } \\
\text { already presented in previous } \\
\text { systematic reviews. }\end{array}$ & $\begin{array}{l}\text { Training vs no training/or } \\
\text { general care }\end{array}$ & $\begin{array}{l}\text { ALSFR/ Medical Outcomes Survey 36-item/QoL: SF-36/Life } \\
\text { satisfaction and well-being/visual analogue scales ROM/Muscle } \\
\text { strength and shortness/Grip strength/Functional activities-Modified } \\
\text { Norris Limb Scale/Muscle strength measured with Chatillon push-pull } \\
\text { gauge/Survival }\end{array}$ \\
\hline Ferreira et al. (35) & $3 \mathrm{RCTs}$ & 63 & Not reported & $\begin{array}{l}\text { Comparison with controls } \\
\text { who had not received RMT } \\
\text { full time or were receiving } \\
\text { training without load }\end{array}$ & $\begin{array}{l}\text { Ventilatory function FVC, FEV1, MVV/Respiratory muscle strength, } \\
\text { MEP and MIP)/Functional capacity, } 6 \mathrm{MWT}\end{array}$ \\
\hline Quinn and Busse (38) & $\begin{array}{l}4 \text { studies with different } \\
\text { designs: before/after } \\
\text { design }(N=1) \text {, single } \\
\text { case }(N=1) \text {, } \\
\text { observational }(N=1) \text {, } \\
\text { RCT }(N=1)\end{array}$ & 63 & $\begin{array}{l}\text { Male: female }=17: 23 \text { (only reported in } 1 \\
\text { study - Zinzi et al. (48)). Age not reported } \\
\text { except for one single case study = one } \\
\text { male, } 49 \text { years old }\end{array}$ & $\begin{array}{l}\text { Healthy controls/healthy } \\
\text { matched controls/usual } \\
\text { care (pharmacological) }\end{array}$ & $\begin{array}{l}\text { Range of motion/Flexibility/Strength/Co-ordinated and reciprocal } \\
\text { movement/Standing, one foot and kneeling balance/Breathing volume } \\
\text { and control/SF-36/Number of falls/Modified falls scale/Berg Balance } \\
\text { Scale/Self' paced/Fast paced gait speed/UHDRS/Physical } \\
\text { examinations of posture/Zung depression scale/MMSE/Barthel Index } \\
\text { (ADL)/Tinetti scale (balance)/PPT/Rehabilitation evaluation scale } \\
\text { (REHAB)/BMD/Interact (behavior assessment)/HR/BP/RR/SHRS }\end{array}$ \\
\hline Fritz et al. (39) & $\begin{array}{l}2 \text { Observational } \\
\text { (without control), } 6 \\
\text { RCTs, } 7 \text { Pre-Post } \\
\text { control group studies, } 2 \\
\text { Pseudo RCT, } 1 \text { single } \\
\text { case study }\end{array}$ & 435 & $\begin{array}{l}\text { Male }=47.25 \% \text { Mean Age }(\mathrm{Y}): \\
\text { range } 28-57\end{array}$ & $\begin{array}{l}\text { Usual care }(N=2) \text {, no } \\
\text { progression in resistance } \\
\text { training }(N=1) \text {, sham }(N= \\
\text { 1), otherwise not specified }\end{array}$ & $\begin{array}{l}\text { Balance/Fitness (cardiovascular function)/Goal attainment/Motor } \\
\text { function and performance/Muscle strength/Number of falls/Physical } \\
\text { activity/Pulmonary function/Rate of chest infections/Ulcer } \\
\text { staging/Spatiotemporal and kinematic parameters of gait and } \\
\text { balance/Walking ability and endurance/Outcome measures of cognitive } \\
\text { function included cognition and psychological measures (depression, } \\
\text { anxiety, and apathy) }\end{array}$ \\
\hline Koopman et al. (31) & $\begin{array}{l}3 \text { RCTs (2 included in } \\
\text { the scoping review) }\end{array}$ & 120 & $\begin{array}{l}\text { One study was conducted in elderly } \\
\text { people (no details provided). No details are } \\
\text { reported in the other studies }\end{array}$ & No treatment or usual care & $\begin{array}{l}\text { Self perceived activity limitations (e.g., Physical Component Summary } \\
\text { of the SF-36 PCS/Physical mobility category of the Nottingham Health } \\
\text { Profile)/Muscle strength/Muscle endurance fatigue/Pain/Adverse events } \\
\text { subdivided into minor adverse events and serious adverse events }\end{array}$ \\
\hline Trujillo-Martín et al. (40) & $\begin{array}{l}1 \text { Clinical trial (pre-post } \\
\text { design) }\end{array}$ & 87 & Mean age $(Y),(S D)=38.1(10.9)$ & No controls & $\begin{array}{l}\text { Neurological examination using the Romberg's Test and a coordination } \\
\text { test with a computer }\end{array}$ \\
\hline
\end{tabular}




\begin{tabular}{|c|c|c|c|c|c|}
\hline References & $\begin{array}{l}\text { Research designs of } \\
\text { included studies }\end{array}$ & $\begin{array}{l}\text { Number of } \\
\text { participants }\end{array}$ & Participant characteristics & Controls & Outcome measures \\
\hline Fonteyn et al. (41) & $\begin{array}{l}14 \text { prospective clinical } \\
\text { trials ( } 4 \text { moderate } \\
\text { quality i.e., comparative } \\
\text { studies }-1 \text { on } \\
\text { cerebellar ataxia). }\end{array}$ & 84 & Not reported & $\begin{array}{l}\text { Controls were patients } \\
\text { receiving treatments later or } \\
\text { not specified }\end{array}$ & $\begin{array}{l}\text { Balance/gait/muscle strength/range of motion/ataxia severity/fall } \\
\text { frequency/gait speed/ADL/FIM/Barthel Incapacitation scores/Hamilton } \\
\text { Rating Scale for Depression/WHOQOL-BREF/NESSCA/SARA }\end{array}$ \\
\hline Marquer et al. (43) & $\begin{array}{l}19 \text { studies including } \\
\text { MS and traumatic } \\
\text { causes. In this scoping } \\
\text { review only } 4 \text { were } \\
\text { included: } 1 \text { RCT and } 3 \\
\text { observational studies. } \\
\text { However, } 3 \text { of these } \\
\text { were already included } \\
\text { in Fonteyn et al. (41). } \\
\text { Only Foltz and Sinaki } \\
\text { (49) is described in the } \\
\text { scoping review. }\end{array}$ & 19 & Not reported & No controls & Subjective self-evaluation of balance \\
\hline Milne et al. (42) & $\begin{array}{l}4 \text { RCTs } 1 \text { Pseudo-RCT } \\
4 \text { Interrupted time } \\
\text { series without a parallel } \\
\text { control group } 5 \\
\text { Case series }\end{array}$ & $\begin{array}{l}292 \text { in total }(21 \\
\text { were not adults) }= \\
271\end{array}$ & $\begin{array}{l}\text { Mean age range (Y): 23.3-62.5 A total of } \\
228 \text { participants (out of 292) were } \\
\text { ambulant, and } 72 \text { were non-ambulant. In } 2 \\
\text { studies, ambulation status was } \\
\text { not reported }\end{array}$ & $\begin{array}{l}\text { No controls or } \\
\text { pharmacological } \\
\text { management alone or } \\
\text { control } \\
\text { group completing verbal } \\
\text { health education and upper } \\
\text { limb exercises (compared to } \\
\text { a cycling regime) or a } \\
\text { control group receiving } \\
\text { sham vibration over the } \\
\text { same duration (compared to } \\
\text { stochastic vibration) }\end{array}$ & $\begin{array}{l}\text { SARA/FIM/Gait speed/ cadence/ FAC/ Number of falls/ICARS (8 } \\
\text { items)/10 mWT/ Gait speed/ Standing capacity/ Spread of feet/ Body } \\
\text { sway/ Knee to tibia test/ Action tremor/ } \\
\text { SF-36/EQ-5D/EQ-VAS/ABC/SCAF//NAS/GAS/BBS/Kinematic and } \\
\text { kinetic gait parameters/Static balance test/ Dynamic balance } \\
\text { acceleration treadmill task/DGI/ TUG/ FRT/ ABC/ Sway amplitude/ } \\
\text { Spatiotemporal/ gait parameters/ FES-I LOS/ SOT/CoP area of 95\% } \\
\text { confidence/Ellipse CoP sway path/ CoP mean velocity/ Barthel } \\
\text { WHOQOL-BREF/ MBI/ 5-item Barthel Index/ Obstacle avoidance task } \\
\text { on a treadmill/ EFAP obstacle subtask/ Sway area }\end{array}$ \\
\hline Hajar and Cooper (44) & $\begin{array}{l}2 \text { quasi randomized } \\
\text { controlled trials }\end{array}$ & $\begin{array}{l}38 \text { (two studies } \\
\text { based on the } \\
\text { same sample) }\end{array}$ & Not reported & Balance exercises only & $\begin{array}{l}\text { Kinematic gait measures (stance time, swing time, and step } \\
\text { length)/2.4-m walk test/Timed "Up \& Go" Test/Vertical Gaze Fixation } \\
\text { Score/Gaze Error Index }\end{array}$ \\
\hline
\end{tabular}


TABLE 1 | Continued

\begin{tabular}{|c|c|c|c|c|c|}
\hline References & $\begin{array}{l}\text { Research designs of } \\
\text { included studies }\end{array}$ & $\begin{array}{l}\text { Number of } \\
\text { participants }\end{array}$ & Participant characteristics & Controls & Outcome measures \\
\hline Intiso et al. (8) & $\begin{array}{l}6 \text { case reports, } 3 \text { case } \\
\text { series, one } \\
\text { case-control study, one } \\
\text { quasi randomized trial } \\
\text { and one randomized } \\
\text { controlled trial }\end{array}$ & 88 & $\begin{array}{l}\text { Gender (number of F/M), information only } \\
\text { in } 3 \text { studies: Case series } 1: 6 / 2 \text { Case } \\
\text { series 2: } 3 / 7 \text { Case series 3: } 2 / 3\end{array}$ & $\begin{array}{l}\text { Balance exercises only } \\
\text { [same studies as in (44)] }\end{array}$ & $\begin{array}{l}\text { BBS/ ABC Scale/Sharpened Romberg Test/ FRT/360 turns/ TUG test/ } \\
6 \text {-MWT/10-WMT/15.2-meter walk test/8-foot (2.4-me) walk } \\
\text { test/5-step test/Balance and gait parameters/ABF device/Static and } \\
\text { dynamic baropodometry/Computerized systems including the } \\
\text { GAITRite system/3D-GA/Force platforms/PSPRS/UPDRS }\end{array}$ \\
\hline
\end{tabular}

6 MWT, Six Minute Walking Test; RM, Repetition Maximum; VO2 max, Maximal Oxygen uptake; 6 MWD, Six Minute Walking Distance; ICU, Intensive Care Unit; CIS-fatigue, Checklist Individual Strength; MVC, Maximum Voluntary Contraction: MVIC, Maximum Voluntary Isokinetic Strength: PIPPS, Perceived Impact of Problem Profile Scale; ESS, Environmental Status Scale; CK, Serum creatine kinase; ALS, Amyotrophic Lateral Sclerosis; ALS-FRS, Amyotrophic Lateral Sclerosis Functional Rating Scale; EQ-VAS, EQ-Visual Analogue Scale; DGI, Dynamic Gait Index; LGMD1. Limb-girdle muscular dystrophies autosomal dominant; LGMD2, Limb-girdle muscular dystrophies autosomal recessive; Lateral Sclerosis Functional Rating Scale; EQ-VAS, EQ-Visual Analogue Scale; DGl, Dynamic Gait Index; LGMD1, Limb-girdle muscular dystrophies autosomal dominant; LGMD2, Limb-girdle muscular dystrophies autosomal recessive;
MND, Motor Neurone Disease; RCT, Randomized Controlled Trial; PNF, Proprioceptive Neuromuscular Facilitation; SRV, Systematic Review; ALSFRS, Amyotrophic Lateral Sclerosis Functional Rating Scale; MT, Manual Muscle strength Testing; FSS, Fatigue Severity Scale; FIM, Functional Independence Measure; FVC, Forced Vital Capacity; BIPAP, Biphasic Positive Airway Pressure; MIP, Maximum Inspiratory Pressure; MEP, Maximal Expiratory Pressure; HRSD, Hamilton Rating Scale for Depression; ESS, Environmental Status Scale; EQ-5D, Health Status questionnaire; SF-36, Short Form-36 Health Survey; SNIP, Sniff Nasal Inspiratory Pressure; RMT, Respiratory Muscle Training; FEV1, Forced Expiratory Volume; MW, Maximum Voluntary Ventilation; QoL, Quality of Life; CMTES, Charcot-Marie-Tooth; Examination Score; FITT, Frequency-Intensity-Time-Type; ROM, Range of motion; BMI, Body mass Index; FFM, Fat Free Mass; RMS, Root Mean Square; PCI, Physiological Cost Index; MHC, Myosin Heavy Chain; VAS, Visual Analogue Scale; METS, Metabolic Equivalent of Task; UHDRS, Unified Huntington's Disease Rating Scale; MMSE, Mini Mental Status Examination: PPT, Physical Performance Test; REHAB, Rehabilitation Evaluation Scale; BMD, Behavior and Mood Disturbance scale; HR, Heart Rate; BP, Blood pressure; RR, Respiratory Rate; SHRS, St Hans rating scale; ADL, Activity Daily Living: LOSWHOQOL-BREF. World Health Organization Quality of Life questionnaire; NESSCA, Examination Score for Spinocerebellar Ataxia; SARA score, Scale for Assessment and Rating of Ataxia; FAC, Functional Ambulation Classification; ICARS, International Cooperative Ataxia Rating Scale; ABC, Activities specific Balance Confidence; SCAFI, Spinocerebellar Functional Index; INAS, Inventory of Non-Ataxia Signs; GAS, Goal Attainment Score; BBS, Berg Balance Scale; TUG, Timed up-and-go test; FES, Falls Self-Efficacy Scale; LOS, Limits of stability; SOT, Sensory Organization Test; WHOQOL-BREF, WHO Quality of Life-BREF; EFAP, Emory Functional Ambulation Profile; RAM, Rotterdam Activity Monitor; FIS, Fatigue Impact Scale; HADS, Hospital Anxiety and Depression Scale; RHS, Rotterdam Handicap Scale; MMT, Manual muscle testing; DASS-21, Depression, Anxiety; Stress scale (short form); PIPP, Perceived Impact of Problem Profile; PEFR, Expected Peak Expiratory Flow Rate; FEV1, Forced Vital Capacity in 1 s; HRQoL, Health-related Quality of Life; MBI, Modified Barthel Index; MRS, Modified Rankin Scale; HDS, Hughes Disability Scale; NHP, Nottingham Health Profile; BI, Barthel Index; HAS, Handicap Assessment Scale; ALSSS, Amyotrophic Lateral Sclerosis Severity Scale; CSI, Caregiver Strain Index; BBS, Berg Balance Scale; ABC, Scale Activities-specific Balance Confidence; FRT, Functional Reach Test; 10-WMT, Ten-Meter Walk Test; ABF, Audio-biofeedback device; 3D-GA, 3D-Gait Analysis; PSPRS, Progressive Supranuclear Palsy Rating Scale; UPDRS, Unified Parkinson's Disease

Rating Scale; FSHD, Facioscapulohumeral Muscular Dystrophy; CoP, Centre of Pressure; OMs, Outcome Measures; FITT FDI, frequency, duration, and intensity score for the Phone-FITT scale. 
TABLE 2 | Mapping to World Health Organisation international classification of function ICF) domains.

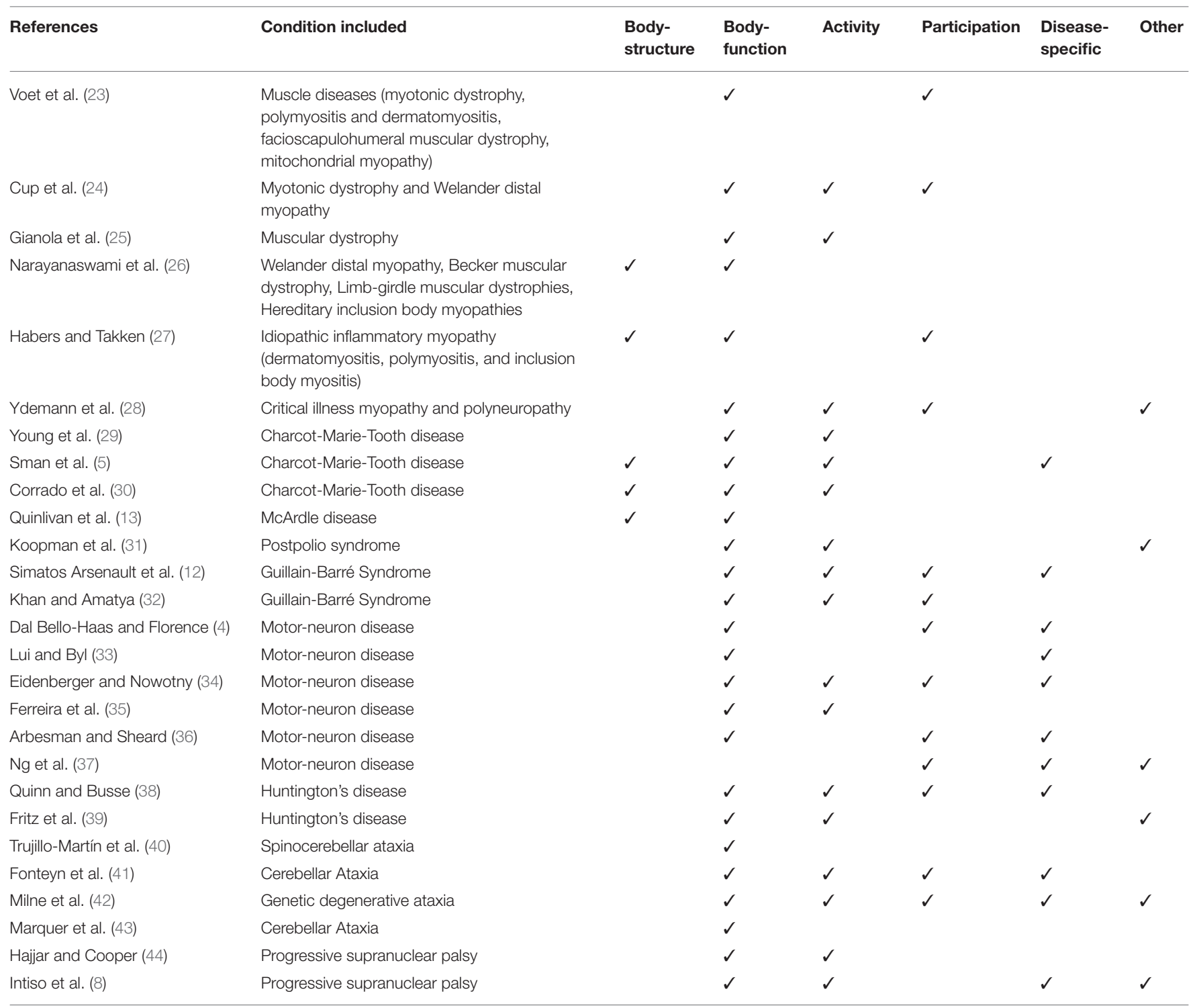

Step One and has been used in one RNC disease group and other neurological conditions (50). Three constructs (i.e., enjoyment, motivation and confidence) are important predictors of physical activity behavior and not assessed within any of the Ox-PAQ items but are relate highly to self-efficacy. The Sources of Self-Efficacy for Physical Activity (52) was thus identified as an additional secondary outcome able to reflect these constructs. Other self-efficacy scales found in the search were specific to particular diseases and populations but did not include neurological conditions. It is important to note that these outcomes were not only reflective of that which is important to stakeholders but also additionally are able to provide mechanistic insight for researchers.

\section{Step Three: Quality and Feasibility Assessment of Proposed Outcome Measurement Instruments}

The OxPAQ questionnaire is a short, 23-item, patient-reported outcome measure, that has been specifically developed for crossdisease application and validated in three long term neurological conditions (MND, Parkinson's disease, Multiple Sclerosis) (50). It was developed using patient interviews and expert reviews and has a manual and online scoring. The Ox-PAQ reports on three domains, Routine Activities (14 items), Emotional Well-Being (5 items) and Social Engagement (4 items). Routine Activities assesses individuals' capacity to engage in regular activities that form the basis of daily life. Emotional Well-Being provides an indication of current mental health status, while 
Social Engagement assesses whether individuals can maintain relationships, both personal and from a wider community perspective. Internal reliability is high (Cronbach's $\alpha$ 0.810.96 ) and validity was demonstrated against relevant domains of the MOS SF-36 and the EQ-5D-5L (50). Sources of SelfEfficacy for Physical Activity is an 18-item questionnaire that measures six aspects (3 items for each source) of self-efficacy for physical activity, specifically: mastery experience, vicarious experience, verbal persuasion by others, self-persuasion, negative affective states and positive affective states (52). Items were pooled from prior qualitative studies, scales of feelings induced by physical activity and sources of self-efficacy more generally. It was refined in a study of 1,406 German adults through principal axis analysis with inter-related factors and confirmatory factor analysis. It is a reliable (Cronbach's $\alpha$ 0.75-0.93), valid (convergent and discriminant).It has not been validated in neurological populations, but the scale was designed to be generally inclusive allowing it to be applied across conditions and populations (52). Other self-efficacy scales target specific conditions and were not generalizable or applicable to people with RNC.

\section{Step Four: Reaching Consensus on the Proposed Core Outcome Set}

Following broad group communication and discussions and a final face to face consensus procedure involving small group discussions, it was agreed that the Ox-PAQ and the Sources of Self-Efficacy for Physical Activity measure should be assessed in trials evaluating physical activity interventions across RNCs given the ways in which they matched the domains and constructs of importance identified by the stakeholder group. This was a group decision by people living with RNC, charity representatives and the research team at the final workshop.

\section{DISCUSSION}

Physical activity research trials in RNCs to date have typically involved targeted exercise intervention and evaluation at the specific disease level despite these diseases leading to variable but similar impairments and functional impacts (for example fatigue, muscle weakness, balance problems, falls and difficulty walking). Our scoping review highlighted the prevalence of interventions, mainly focusing on structured exercise and typically underpinned by standard approaches (53) highlighting the role of physical activity and exercise as a critical enabler of participation for all those living with common and rarer long term neurological diseases (54). Our scoping review of the literature identified outcome measures appropriate for the specific body structure, function and activity level changes targeted by these interventions, but there was a degree of mismatch between these outcomes and constructs identified as important to people living with RNCs (e.g., assessments that capture changes at the level of participation).

We utilized a person-centered approach leading to the proposal of a meaningful core outcome measurement set for use when researching physical activity interventions for people living with RNCs. Our collaborative and participatory design involved members of the public, including people living with RNCs, representatives of charities and support groups for RNCs and is the first core outcome set to our knowledge which has specifically focused on physical activity interventions for RNCs. Stakeholder engagement is receiving increasing recognition in patient-reported outcomes research (18) and clinical trials $(55,56)$ so as to ensure that interventions and outcomes are relevant to the target populations. A core outcome set for disease modification trials for dementia has been developed with stakeholder input and involvement of the research community. This was achieved through a number of stages, including a systematic review of outcome measures, a consultation with patient and public involvement representatives and a final consensus reached with the dementia research community (20). A similar approach was used to develop a core outcome measure set for exercise studies in Multiple Sclerosis (57), where a group consisting of experts in the field, support group representatives and expert patients, jointly discussed a predefined core set for Multiple Sclerosis. This was based on the World Health Organisation International Classification of Function and included body structure and function, activity and participation categories. Our approach differed somewhat in that we initially elicited discussion and reflection from our stakeholder groups on the domains considered important when engaging in physical activity interventions, but without presenting any work undertaken in previous studies.

Outcomes identified in the scoping review assessed the effect of physical activity interventions primarily at the level of body functions and structures, functional activities. There were fewer identified outcomes at participation level, in contrast to the domains prioritized by our stakeholder group, namely physical and psychological well-being and participation to day-to-day activities. In the scoping review, measures of quality of life and health-related well-being were identified, but these did not (in the views of our stakeholder group) sufficiently capture the breadth of areas of importance in relation to participation and physical activity in RNCs. For example, the 36-Item Short Form Survey is more focused on levels of vigorous and moderate activities, rather than independence in day-to-day activities. The Ox-PAQ and the Sources of Self-Efficacy for Physical Activity measure were however considered to reflect meaningful outcomes of physical activity interventions for people with RNCs.

Whilst the identified and proposed outcomes are clearly relevant to people with RNC, it is not yet clear how well the measures perform within and between these populations nor whether they fully capture that which is meaningful to people with RNCs. For example, the Sources of Self-Efficacy Scale may not fully capture enjoyment for physical activity; it may be that a purpose developed enjoyment scale $(58,59)$ is more appropriate in different settings. The broad range of rare neurological diseases where physical activity interventions are indicated are a specific challenge. A key limitation is that we did not consistently have stakeholders present at all workshops with faster progressing conditions, those with significant cognitive disorders or carers, relying on the charity representatives to bring accounts of these experiences. People were invited, but the additional complexity 
of those conditions may have affected engagement in all steps. Future validation work will need to include these groups to inform the implementation of the proposed core outcome set.

\section{CONCLUSION}

We propose a core outcome set, developed in collaboration with people living with RNC and their representatives, for use in studies of physical activity interventions. The two measures proposed were selected to include domains of importance to people living with these diseases.

\section{DATA AVAILABILITY STATEMENT}

The original contributions presented in the study are included in the article/supplementary material, further inquiries can be directed to the corresponding author/s.

\section{ETHICS STATEMENT}

Ethical review and approval was not required for the study on human participants, in accordance with the local legislation and institutional requirements.

\section{AUTHOR CONTRIBUTIONS}

GR and MB: conception and organization of the research project, design and review and critique of the analysis, and writing of the first draft and review and critique of the manuscript. VB: organization of the research project, design and review and critique of the analysis, and writing of the first draft and review

\section{REFERENCES}

1. Richter T, Nestler-Parr S, Babela R, Khan ZM, Tesoro T, Molsen $\mathrm{E}$, et al. Rare disease terminology and definitions-a systematic global review: report of the ISPOR rare disease special interest group. Value Health J Int Soc Pharmacoeconomics Outcomes Res. (2015) 18:90614. doi: 10.1016/j.jval.2015.05.008

2. Angelis A, Tordrup D, Kanavos P. Socio-economic burden of rare diseases: a systematic review of cost of illness evidence. Health Policy Amst Neth. (2015) 119:964-79. doi: 10.1016/j.healthpol.2014.12.016

3. WHO. International Classification of Functioning, Disability Health (ICF). WHO. World Health Organization. Available online at: http://www.who.int/ classifications/icf/en/ (accessed September 2, 2020).

4. Dal Bello-Haas V, Florence JM. Therapeutic exercise for people with amyotrophic lateral sclerosis or motor neuron disease. Cochrane Database Syst Rev. (2013) CD005229. doi: 10.1002/14651858.CD005229.pub3

5. Sman AD, Hackett D, Fiatarone Singh M, Fornusek C, Menezes MP, Burns J. Systematic review of exercise for Charcot-Marie-Tooth disease. J Peripher Nerv Syst JPNS. (2015) 20:347-62. doi: 10.1111/jns.12116

6. Collett J, Dawes H, Bateman J, Dawes H, Bateman J. Physical Activity for Long Term Neurological Conditions : Multiple Sclerosis and Huntington's Disease. Clinical Exercise Science. (2016). p. 155-77. Available online at: https://www. taylorfrancis.com/ (accessed June 21, 2020).

7. Quinn L, Kegelmeyer D, Kloos A, Rao AK, Busse M, Fritz NE. Clinical recommendations to guide physical therapy practice for Huntington disease. Neurology. (2020) 94:217-28. doi: 10.1212/WNL.0000000000008887 and critique of the manuscript. HD: conception and organization of the research project, design of the analysis, and writing of the first draft and review and critique of the manuscript. $\mathrm{AB}, \mathrm{TJ}, \mathrm{JM}, \mathrm{LP}, \mathrm{RP}, \mathrm{MR}$, and LR: conception of the research project, design of the analysis, and review and critique of the manuscript. FJ and ER: conception and organization of the research project, design of the analysis, and review and critique of the manuscript. All authors contributed to the article and approved the submitted version.

\section{FUNDING}

This work was funded by an NIHR Programme Development Grant RP-DG-0517-10002 (Co-Chief Investigators: GR and MB). This is a summary of independent research funded by the National Institute for Health Research (NIHR)'s Programme Development Grant Programme. Centre for Trials Research receives funding from Health and Care Research Wales and Cancer Research UK. Open Access funding has been made available from Cardiff University.

\section{ACKNOWLEDGMENTS}

We would like the acknowledge the input of our stakeholder groups and charity representatives from the Muscular Dystrophy UK, Ataxia UK, Hereditary Spastic Paraparesis support group, Progressive Supranuclear Palsy Association, Huntington's Disease Association of England and Wales, Multiple Systems Atrophy Trust, Motor Neuron Disease Association. The scoping review that formed part of this work was registered on the Open Science Framework https://osf.io/4cr32/.

8. Intiso D, Bartolo M, Santamato A, Di Rienzo F. The role of rehabilitation in patients with progressive supranuclear palsy: a narrative review. $P M R$. (2018) 10:636-45. doi: 10.1016/j.pmrj.2017.12.011

9. WHO. Global Recommendations on Physical Activity for Health. WHO. Available online at: http://www.who.int/dietphysicalactivity/factsheet_ recommendations/en/index.html (accessed August 29, 2021).

10. Elsworth C, Winward C, Sackley C, Meek C, Freebody J, Esser P, et al. Supported community exercise in people with long-term neurological conditions: a phase II randomized controlled trial. Clin Rehabil. (2011) 25:588-98. doi: 10.1177/0269215510392076

11. Wallace A, Pietrusz A, Dewar E, Dudziec M, Jones K, Hennis $\mathrm{P}$, et al. Community exercise is feasible for neuromuscular diseases and can improve aerobic capacity. Neurology. (2019) 92:e1773-85. doi: 10.1212/WNL.0000000000007265

12. Simatos Arsenault N, Vincent P-O, Yu BHS, Bastien R, Sweeney A. Influence of exercise on patients with Guillain-Barré syndrome: a systematic review. Physiother Can. (2016) 68:367-76. doi: 10.3138/ptc.2015-58

13. Quinlivan R, Vissing J, Hilton-Jones D, Buckley J. Physical training for McArdle disease. Cochrane Database Syst Rev. (2011) CD007931. doi: 10.1002/14651858.CD007931.pub2

14. Heneghan C, Goldacre B, Mahtani KR. Why clinical trial outcomes fail to translate into benefits for patients. Trials. (2017) 18:122. doi: 10.1186/s13063-017-1870-2

15. Williamson P, Altman D, Blazeby J, Clarke M, Gargon E. Driving up the quality and relevance of research through the use of agreed core outcomes. J Health Serv Res Policy. (2012) 17:1-2. doi: 10.1258/jhsrp.2011.011131 
16. Clarke M. Standardising outcomes for clinical trials and systematic reviews. Trials. (2007) 8:39. doi: 10.1186/1745-6215-8-39

17. Dworkin RH, Turk DC, Farrar JT, Haythornthwaite JA, Jensen MP, Katz NP, et al. Core outcome measures for chronic pain clinical trials: IMMPACT recommendations. Pain. (2005) 113:9-19. doi: 10.1016/j.pain.2004.09.012

18. Devane D, Begley CM, Clarke M, Horey D, OBoyle C. Evaluating maternity care: a core set of outcome measures. Birth Berkeley Calif. (2007) 34:16472. doi: 10.1111/j.1523-536X.2006.00145.x

19. Moore JL, Potter K, Blankshain K, Kaplan SL, O’Dwyer LC, Sullivan JE. A core set of outcome measures for adults with neurologic conditions undergoing rehabilitation: a clinical practice guideline. J Neurol Phys Ther. (2018) 42:174. doi: 10.1097/NPT.0000000000000229

20. Webster L, Groskreutz D, Grinbergs-Saull A, Howard R, O’Brien JT, Mountain G, et al. Development of a core outcome set for disease modification trials in mild to moderate dementia: a systematic review, patient and public consultation and consensus recommendations. Health Technol Assess. (2017) 21:1-192. doi: 10.3310/hta21260

21. Guideline for Selecting Instruments for a Core Outcome Set • COSMIN. COSMIN. Available online at: https://www.cosmin.nl/tools/guidelineselecting-proms-cos/ (accessed March 18, 2021).

22. COSMIN Taxonomy of Measurement Properties. Available online at: https:// www.cosmin.nl/tools/cosmin-taxonomy-measurement-properties/ (accessed August 29, 2021).

23. Voet NBM, van der Kooi EL, Riphagen II, Lindeman E, van Engelen BGM, Geurts ACH. Strength training and aerobic exercise training for muscle disease. Cochrane Database Syst Rev. (2013) CD003907. doi: 10.1002/14651858.CD003907.pub4

24. Cup EHC, Sturkenboom IHWM, Pieterse AJ, Hendricks HT, van Engelen BGM, Oostendorp RAB, et al. The evidence for occupational therapy for adults with neuromuscular diseases: a systematic review. OTJR Occup Particip Health. (2008) 28:12-8. doi: 10.3928/15394492-20080101-02

25. Gianola S, Pecoraro V, Lambiase S, Gatti R, Banfi G, Moja L. Efficacy of muscle exercise in patients with muscular dystrophy: a systematic review showing a missed opportunity to improve outcomes. PLoS ONE. (2013) 8:e65414. doi: 10.1371/journal.pone.0065414

26. Narayanaswami P, Weiss M, Selcen D, David W, Raynor E, Carter G, et al. Evidence-based guideline summary: diagnosis and treatment of limb-girdle and distal dystrophies: report of the guideline development subcommittee of the American Academy of Neurology and the practice issues review panel of the American Association of Neuromuscular \& Electrodiagnostic Medicine. Neurology. (2014) 83:1453-63. doi: 10.1212/WNL.0000000000000892

27. Habers GEA, Takken T. Safety and efficacy of exercise training in patients with an idiopathic inflammatory myopathy-a systematic review. Rheumatol Oxf Engl. (2011) 50:2113-24. doi: 10.1093/rheumatology/ker292

28. Ydemann M, Eddelien HS, Lauritsen AØ. Treatment of critical illness polyneuropathy and/or myopathy - a systematic review. Dan Med J. (2012) 59:A4511. Available online at: https://ugeskriftet.dk/dmj/treatmentcritical-illness-polyneuropathy-and-or-myopathy-systematic-review

29. Young P, De Jonghe P, Stögbauer F, Butterfass-Bahloul T. Treatment for Charcot-Marie-Tooth disease. Cochrane Database Syst Rev. (2008) CD006052. doi: 10.1002/14651858.CD006052.pub2

30. Corrado B, Ciardi G, Bargigli C. Rehabilitation management of the CharcotMarie-Tooth syndrome: a systematic review of the literature. Medicine. (2016) 95:e3278. doi: 10.1097/MD.0000000000003278

31. Koopman FS, Beelen A, Gilhus NE, de Visser M, Nollet F. Treatment for postpolio syndrome. Cochrane Database Syst Rev. (2015) CD007818. doi: 10.1002/14651858.CD007818.pub3

32. Khan F, Amatya B. Rehabilitation interventions in patients with acute demyelinating inflammatory polyneuropathy: a systematic review. Eur J Phys Rehabil Med. (2012) 48:507-22. Available online at: https://www. minervamedica.it/en/journals/europa-medicophysica/article.php?cod= R33Y2012N03A0507

33. Lui AJ, Byl NN. A systematic review of the effect of moderate intensity exercise on function and disease progression in amyotrophic lateral sclerosis. J Neurol Phys Ther JNPT. (2009) 33:68-87. doi: 10.1097/NPT.0b013e31819912d0

34. Eidenberger M, Nowotny S. Inspiratory muscle training in patients with amyotrophic lateral sclerosis: a systematic review. NeuroRehabilitation. (2014) 35:349-61. doi: 10.3233/NRE-141148
35. Ferreira GD, Costa ACC, Plentz RDM, Coronel CC, Sbruzzi G. Respiratory training improved ventilatory function and respiratory muscle strength in patients with multiple sclerosis and lateral amyotrophic sclerosis: systematic review and meta-analysis. Physiotherapy. (2016) 102:221-8. doi: 10.1016/j.physio.2016.01.002

36. Arbesman M, Sheard K. Systematic review of the effectiveness of occupational therapy-related interventions for people with amyotrophic lateral sclerosis. Am J Occup Ther. (2014) 68:20-6. doi: 10.5014/ajot.2014.008649

37. Ng L, Khan F, Mathers S. Multidisciplinary care for adults with amyotrophic lateral sclerosis or motor neuron disease. Cochrane Database Syst Rev. (2009) CD007425. doi: 10.1002/14651858.CD007425.pub2

38. Quinn L, Busse M. Physiotherapy clinical guidelines for Huntington's disease. Neurodegener Dis Manag. (2012) 2:21-31. doi: 10.2217/nmt.11.86

39. Fritz NE, Rao AK, Kegelmeyer D, Kloos A, Busse M, Hartel L, et al. Physical therapy and exercise interventions in Huntington's disease: a mixed methods systematic review. J Huntingt Dis. (2017) 6:217-35. doi: 10.3233/JHD-170260

40. Trujillo-Martín MM, Serrano-Aguilar P, Monton-Alvarez F, Carrillo-Fumero R. Effectiveness and safety of treatments for degenerative ataxias: a systematic review. Mov Disord. (2009) 24:1111-24. doi: 10.1002/mds.22564

41. Fonteyn EMR, Keus SHJ, Verstappen CCP, Schöls L, de Groot IJM, van de Warrenburg BPC. The effectiveness of allied health care in patients with ataxia: a systematic review. J Neurol. (2014) 261:2518. doi: 10.1007/s00415-013-6910-6

42. Milne SC, Corben LA, Georgiou-Karistianis N, Delatycki MB, Yiu EM. Rehabilitation for individuals with genetic degenerative ataxia: a systematic review. Neurorehabil Neural Repair. (2017) 31:609-22. doi: 10.1177/1545968317712469

43. Marquer A, Barbieri G, Pérennou D. The assessment and treatment of postural disorders in cerebellar ataxia: a systematic review. Ann Phys Rehabil Med. (2014) 57:67-78. doi: 10.1016/j.rehab.2014.01.002

44. Hajjar SH, Cooper JK. Progressive supranuclear palsy treatment-A systematic review. Basal Ganglia. (2016) 2:758. doi: 10.1016/j.baga.2016.01.004

45. Drory VE, Goltsman E, Reznik JG, Mosek A, Korczyn AD. The value of muscle exercise in patients with amyotrophic lateral sclerosis. J Neurol Sci. (2001) 191:133-7. doi: 10.1016/s0022-510x(01)00610-4

46. Bello-Haas VD, Florence JM, Kloos AD, Scheirbecker J, Lopate G, Hayes SM, et al. A randomized controlled trial of resistance exercise in individuals with ALS. Neurology. (2007) 68:2003-7. doi: 10.1212/01.wnl.0000264418.92308.a4

47. Dalbello-Haas V, Florence JM, Krivickas LS. Therapeutic exercise for people with amyotrophic lateral sclerosis or motor neuron disease. Cochrane Database Syst Rev. (2008) CD005229. doi: 10.1002/14651858.CD005229.pub2

48. Zinzi P, Salmaso D, De Grandis R, Graziani G, Maceroni S, Bentivoglio A, et al. Effects of an intensive rehabilitation programme on patients with Huntington's disease: a pilot study. Clin Rehabil. (2007) 21:603-13. doi: $10.1177 / 0269215507075495$

49. Folz TJ, Sinaki M. A nouveau aid for posture training in degenerative disorders of the central nervous system. J. Musculoskeletal Pain. (1995) 3:5970. doi: 10.1300/J094v03n04_07

50. Morley D, Dummett S, Kelly L, Jenkinson C. Measuring improvement in health-status with the Oxford Participation and Activities Questionnaire (Ox-PAQ). Patient Relat Outcome Meas. (2019) 10:153-6. doi: 10.2147/PROM.S198619

51. Morley D, Dummett S, Kelly L, Jenkinson C. Administering the routine activities domain of the Oxford participation and activities questionnaire as a stand-alone scale: the Oxford routine activities measure. Patient Relat Outcome Meas. (2018) 9:239-43. doi: 10.2147/PROM.S160263

52. Warner LM, Schüz B, Wolff JK, Parschau L, Wurm S, Schwarzer R. Sources of self-efficacy for physical activity. Health Psychol. (2014) 33:1298308. doi: 10.1037/hea0000085

53. Garber CE, Blissmer B, Deschenes MR, Franklin BA, Lamonte MJ, Lee I$\mathrm{M}$, et al. American College of Sports Medicine position stand. Quantity and quality of exercise for developing and maintaining cardiorespiratory, musculoskeletal, and neuromotor fitness in apparently healthy adults: guidance for prescribing exercise. Med Sci Sports Exerc. (2011) 43:133459. doi: 10.1249/MSS.0b013e318213fefb

54. Quinn L, Morgan D. From disease to health: physical therapy health promotion practices for secondary prevention in adult and 
pediatric neurologic populations. J Neurol Phys Ther JNPT. (2017) 41:S46-54. doi: 10.1097/NPT.0000000000000166

55. Price A, Albarqouni L, Kirkpatrick J, Clarke M, Liew SM, Roberts N, et al. Patient and public involvement in the design of clinical trials: an overview of systematic reviews. J Eval Clin Pract. (2018) 24:240-53. doi: 10.1111/jep. 12805

56. Brett J, Staniszewska S, Mockford C, Herron-Marx S, Hughes J, Tysall C, et al. Mapping the impact of patient and public involvement on health and social care research: a systematic review. Health Expect Int J Public Particip Health Care Health Policy. (2014) 17:637-50. doi: 10.1111/j.1369-7625.2012. 00795.x

57. Paul L, Coote S, Crosbie J, Dixon D, Hale L, Holloway E, et al. Core outcome measures for exercise studies in people with multiple sclerosis: recommendations from a multidisciplinary consensus meeting. Mult Scler Houndmills Basingstoke Engl. (2014) 20:1641-50. doi: 10.1177/1352458514526944

58. Mullen SP, Olson EA, Phillips SM, Szabo AN, Wójcicki TR, Mailey EL, et al. Measuring enjoyment of physical activity in older adults: invariance of the physical activity enjoyment scale (paces) across groups and time. Int J Behav Nutr Phys Act. (2011) 8:103. doi: 10.1186/1479-58688-103

59. Kendzierski D, DeCarlo KJ. Physical activity enjoyment scale: two validation studies. J Sport Exerc Psychol. (1991) 13:50-64. doi: 10.1123/jsep.13.1.50
Author Disclaimer: The views expressed are those of the authors and not necessarily those of the NHS, the NIHR, or the Department of Health and Social Care.

Conflict of Interest: The authors declare that the research was conducted in the absence of any commercial or financial relationships that could be construed as a potential conflict of interest.

Publisher's Note: All claims expressed in this article are solely those of the authors and do not necessarily represent those of their affiliated organizations, or those of the publisher, the editors and the reviewers. Any product that may be evaluated in this article, or claim that may be made by its manufacturer, is not guaranteed or endorsed by the publisher.

Copyright (c) 2021 Ramdharry, Buscemi, Boaz, Dawes, Jaki, Jones, Marsden, Paul, Playle, Randell, Robling, Rochester and Busse. This is an open-access article distributed under the terms of the Creative Commons Attribution License (CC BY). The use, distribution or reproduction in other forums is permitted, provided the original author(s) and the copyright owner(s) are credited and that the original publication in this journal is cited, in accordance with accepted academic practice. No use, distribution or reproduction is permitted which does not comply with these terms. 